\title{
Estudo do gasto energético por meio da água duplamente marcada: fundamentos, utilização e aplicações
}

\author{
The study of energy expenditure through doubly labeled \\ water: principles, use and applications
}

Fernanda Baeza SCAGLIUSI'

Antonio Herbert LANCHA JÚNIOR²

\section{R E S U M O}

A medida do gasto energético total é fundamental em estudos de nutrição e metabolismo. A água duplamente marcada é uma forma de calorimetria indireta que mede precisa e acuradamente o gasto energético total de indivíduos fora de confinamento. Esse método já foi aplicado com diversas finalidades, como medir a demanda energética decorrente de doenças crônicas e da atividade física. Além disso, também foi usada em estudos sobre as conseqüências da desnutrição e a etiologia da obesidade, especialmente demonstrando as diferenças inter-raciais quanto ao gasto energético total. Tal técnica também pode ser utilizada para validar outros métodos de avaliação da prática de atividade física e/ou do gasto energético total. Embora diversos estudos de validação tenham sido conduzidos, nenhum método demonstrou bom desempenho nas estimativas individuais. Finalmente, a água duplamente marcada tem sido amplamente empregada em países desenvolvidos como marcador do consumo energético, validando as estimativas obtidas pelos métodos de avaliação do consumo alimentar. Esse uso também deve ser encorajado em países em desenvolvimento, para que se possa considerar o erro das estimativas de ingestão energética dos métodos de avaliação do consumo alimentar, utilizados nas pesquisas de nutrição humana dessas nações.

Termos de indexação: água duplamente marcada, atividade física, gasto energético, obesidade, sub-relato.

\section{A B S T R A C T}

The measurement of total energy expenditure is fundamental in nutritional and metabolic studies. Doubly labeled water is a form of indirect calorimetry, which measures precisely and accurately total energy expenditure

\footnotetext{
1 Bolsista CNPq; Doutoranda, Laboratório de Nutrição e Metabolismo Aplicados à Atividade Motora, Departamento de Biodinâmica do Movimento do Corpo Humano, Escola de Educação Física, Universidade de São Paulo. Av. Prof Mello Moraes, 65, 05508-900, São Paulo, SP, Brasil. Correspondência para/Correspondence to: F.B. SCAGLIUSI. E-mail: <fesc@usp.br>.

2 Departamento de Biodinâmica do Movimento do Corpo Humano, Escola de Educação Física, Universidade de São Paulo. São Paulo, SP, Brasil.
} 
in free-living individuals. This method has been applied for several ends, such as to measure energy expenditure during chronic illness and physical activity. It has also been used in studies concerning the consequences of malnutrition and the etiology of obesity, demonstrating the inter-racial differences in total energy expenditure in particular. The technique can also be used to validate other methods of physical activity assessment and total energy expenditure estimates. Although many validation studies have been conducted, none demonstrated good individual performance. Finally, the doubly labeled water has been widely applied in developed countries as a marker of energy intake, validating the energy intake estimates derived from dietary assessment methods. This application should also be encouraged in developing countries, in order to establish the error associated with energy intake estimates obtained by the dietary assessment methods used in the human nutrition studies of these nations.

Indexing terms: doubly labeled water, physical activity, energy expenditure, obesity, underreporting.

\section{N T R O D U Ç Ã O}

A medida do gasto energético total é um componente fundamental em diversas pesquisas das áreas de metabolismo e nutrição'. No século XVIII, Lavosier e Seguin começaram a estudar a respiração como um processo análogo à combustão. Tais cientistas confinaram animais em câmaras para quantificar seu consumo de oxigênio e sua produção de gás carbônico. A partir desses experimentos, diversos postulados foram determinados e, fundamentalmente, foi estabelecida a metodologia da calorimetria indireta, que continua em voga até os dias atuais ${ }^{1}$. Desde então, as câmaras sofisticaram-se muito e diversos equipamentos auxiliares foram desenvolvidos. Entretanto, por mais sofisticadas que tais câmaras sejam, elas nunca serão capazes de reproduzir o número e a complexidade das tarefas que os indivíduos desempenham rotineiramente em suas vidas.

A técnica da água duplamente marcada permite, por sua vez, medir o gasto energético de indivíduos fora de confinamento, sem causar nenhuma modificação no cotidiano. O advento desse método data de 1949, quando Lifson et al. ${ }^{2}$ administraram água marcada com ${ }^{18} \mathrm{O}$ a animais e demonstraram que o oxigênio do gás carbônico expirado era proveniente da água corporal. Atualmente, sabe-se que isso é o resultado do equilíbrio isotópico entre os átomos de oxigênio da água e do gás carbônico. Em 1955, Lifson et al. ${ }^{3}$ afirmaram que a produção total de gás carbônico poderia ser mensurada pelas diferentes eliminações da água marcada com as formas isotópicas de hidrogênio e oxigênio ou, respectivamente, ${ }^{18} \mathrm{O}$ e ${ }^{2} \mathrm{H}$ (deutério). O deutério é eliminado como água, enquanto que $0{ }^{18} \mathrm{O}$ é eliminado como água e gás carbônico. Assim, a diferença entre tais taxas de eliminação, corrigidas pelo conjunto (pool) de água corporal, corresponderia à produção de gás carbônico, que, por equações de calorimetria indireta, é convertida ao gasto energético total. Matematicamente, essa relação está representada na equação 1 (Quadro 1).

Logicamente, para que a equação 1 possa ser utilizada, é necessário calcular o conjunto de água corporal (representado na equação como N). O espaço de diluição do ${ }^{18} \mathrm{O}$ é bastante semelhante ao conjunto de água corporal total do indivíduo, e pode ser calculado pelas razões entre a abundância isotópica presente na água potável, na dose administrada de água duplamente marcada e no conjunto de água corporal, antes e depois da aplicação dos isótopos ${ }^{4}$.

A equação 1, proposta por Lifson et al. ${ }^{3}$, baseia-se em diversas premissas, que, com o tempo, se mostraram inválidas até certo grau. Entretanto, formas de correção foram desenvolvidas, o que modificou a equação original ${ }^{5}$. As correções mais importantes são quanto ao fracionamento dos isótopos e ao tamanho do $\mathrm{N}$. O material fracionado não carrega consigo a mesma quantidade de marcador, o que complica o modelo original ${ }^{1}$. Atualmente, também se sabe que o hidrogênio e o oxigênio renovam-se em 
outros conjuntos do organismo, que não o de água corporal, e que diferem de tamanho entre si. Schoeller et al. ${ }^{6}$ propuseram que o conjunto de hidrogênio é de 3\% a 5\% maior que o de oxigênio, que, por sua vez, é $1 \%$ maior que o de água. Dessa forma ${ }^{6}$, efetuando-se as correções para o fracionamento e o tamanho do $\mathrm{N}$, a equação foi modificada, gerando a equação 2 , apresentada no Quadro 1. O gasto energético total (GET) pode, então, ser calculado por meio da fórmula de Weir? representada no Quadro 1, como a equação 3.
Deve-se entender que o equivalente energético do $\mathrm{CO}_{2}$ varia bastante, dependendo dos substratos que estão sendo utilizados e, portanto, do quociente respiratório (QR). Em sujeitos em balanço energético, O QR deve ser igual ao quociente alimentar (QA), que corresponde à quantidade calculada de $\mathrm{CO}_{2}$ produzido/oxigênio consumido na oxidação de todos os combustíveis "metabolizáveis" 8 . Black et al. ${ }^{9}$ desenvolveram e validaram uma fórmula de cálculo do QA a partir de inquéritos alimentares, de tal forma que esse

Quadro 1. Equações utilizadas para cálculo do gasto energético total (GET), segundo a técnica da água duplamente marcada.

\begin{tabular}{|c|c|c|c|c|c|}
\hline $\begin{array}{l}\text { Número da } \\
\text { equação }\end{array}$ & $\begin{array}{c}\text { Fórmula } \\
\text { matemática }\end{array}$ & Legenda & Observações & Finalidade & Referências \\
\hline 1 & $\begin{array}{l}\mathrm{rCO}_{2}= \\
\mathrm{N} / 2 *(\mathrm{Ko}-\mathrm{Kd})\end{array}$ & $\begin{array}{l}\mathrm{rCO}_{2} \text { : taxa do fluxo de } \\
\mathrm{CO}_{2} \\
\mathrm{~N} \text { : conjunto de água } \\
\text { corporal } \\
\mathrm{Ko} \text { : taxa de eliminação } \\
\text { do }{ }^{18} \mathrm{O} \\
\mathrm{Kd} \text { : taxa de eliminação } \\
\text { do deutério }\end{array}$ & $\begin{array}{l}\text { A divisão do } \mathrm{N} \text { por } 2 \text { re- } \\
\text { flete o fato de que uma } \\
\text { molécula de } \mathrm{CO}_{2} \text { remove } \\
\text { dois átomos de } \mathrm{O}_{2} \text { e uma } \\
\text { molécula de } \mathrm{H}_{2} \mathrm{O} \text { só re- } \\
\text { move um átomo. }\end{array}$ & $\begin{array}{l}\text { Calcular a produção de } \\
\mathrm{CO}_{2} \text { a partir da água du- } \\
\text { plamente marcada. }\end{array}$ & $\begin{array}{l}\text { Lifson et al. }^{3} \\
\text { Speakman }\end{array}$ \\
\hline 2 & $\begin{array}{l}\mathrm{rCO}_{2}=[\mathrm{N} / 2,078 * \\
(1,01 \mathrm{Ko}-1,04 \mathrm{Kd})]- \\
0,0246 r_{\mathrm{GF}}\end{array}$ & $\begin{array}{l}\mathrm{rCO}_{2} \text { : taxa do fluxo de } \\
\mathrm{CO}_{2} \\
\mathrm{~N} \text { : conjunto de água } \\
\text { corporal } \\
\mathrm{Ko} \text { : taxa de eliminação } \\
\text { do }{ }^{18} \mathrm{O} \\
\mathrm{Kd} \text { : taxa de eliminação } \\
\text { do deutério } \\
\mathrm{r}_{\mathrm{GF}} \text { : fator de correção } \\
\text { para o fracionamento } \\
\text { dos isótopos }\end{array}$ & $\begin{array}{l}\mathrm{N}=[(\mathrm{No} / 1,01)+(\mathrm{Nd} / \\
1,04)] / 2 \\
r_{\mathrm{GF}}=1,05 * \mathrm{~N}(1,01 \mathrm{Ko}- \\
1,04 \mathrm{Kd})\end{array}$ & $\begin{array}{l}\text { Idem equação 1, porém } \\
\text { incorporando correções } \\
\text { quanto ao fraciona- } \\
\text { mento de isótopos e às } \\
\text { diferenças de espaço de } \\
\text { diluição dos isótopos. }\end{array}$ & Schoeller et al. ${ }^{6}$ \\
\hline 3 & $\begin{array}{l}\mathrm{GET}=(3,044 * \mathrm{QR}+ \\
1,104) \mathrm{rCO}_{2}\end{array}$ & $\begin{array}{l}\text { GET: gasto energético } \\
\text { total } \\
\text { QR: quociente respira- } \\
\text { tório } \\
\mathrm{rCO}_{2} \text { : taxa do fluxo de } \\
\mathrm{CO}_{2}\end{array}$ & & $\begin{array}{l}\text { Calcular o GET, em } \\
\text { kcal/dia. }\end{array}$ & Weir $^{7}$ \\
\hline 4 & $\begin{array}{l}Q A=(p * 0,81)+ \\
(f * 0,71)+(c * 1,00) \\
+(a * 0,67)\end{array}$ & $\begin{array}{l}\text { QA: quociente ali- } \\
\text { mentar } \\
\text { VET: valor energético } \\
\text { total da dieta } \\
\text { p = energia fornecida } \\
\text { pelas proteínas } N E T \\
f=\text { energia fornecida } \\
\text { pelos lipídeos/NET } \\
c=\text { energia fornecida } \\
\text { pelos carboidratos } N E T \\
\text { a = energia fornecida } \\
\text { pelo álcoolNET }\end{array}$ & $\begin{array}{l}\text { A energia fornecida por } \\
\text { cada componente é cal- } \\
\text { culada multiplicando-se } \\
\text { sua quantidade pelos fa- } \\
\text { tores de Southgate \& } \\
\text { Durnin }{ }^{10}: 4 \text { (proteínas); } 9 \\
\text { (lipídeos); 3,75 (car- } \\
\text { boidratos) e } 7 \text { (álcool). } \\
\text { As constantes apresen- } \\
\text { tadas na fórmula são va- } \\
\text { lores clássicos para o QA } \\
\text { de cada combustível. }\end{array}$ & $\begin{array}{l}\text { Como não é viável medir } \\
\text { o QR em muitos estudos, } \\
\text { esta fórmula calcula o } \\
\text { QA, que deve ser usado } \\
\text { no lugar do QR na equa- } \\
\text { ção 3, para que o gasto } \\
\text { energético total possa ser } \\
\text { calculado. }\end{array}$ & Black et al. ${ }^{9}$ \\
\hline
\end{tabular}


possa ser utilizado no lugar do QR. Deve-se lembrar que a substituição do QR pelo QA diminui a precisão da água duplamente marcada ${ }^{5}$ em $1 \%$ a $2 \%$. A equação ${ }^{10}$ para tal substituição encontra-se no Quadro 1, como equação 4.

As taxas de eliminação dos isótopos podem ser obtidas por meio da metodologia de dois pontos ou de múltiplos pontos. Na primeira, tal taxa é calculada a partir da urina coletada um dia após a administração da dose e no final do período metabólico. Na segunda, são feitas diversas coletas ao longo do período metabólico. A primeira forma obterá um valor correto para o fluxo total entre dois pontos no tempo, mesmo em circunstâncias nas quais ocorram variações sistemáticas que tornem o cálculo da taxa diária do fluxo quase inapropriado. A metodologia de vários pontos, contudo, consegue fornecer uma estimativa da variação em torno da taxa diária de produção de gás carbônico, que reflete tanto os efeitos da variação fisiológica quanto da instrumental ${ }^{11}$. Há pouca literatura que compara a precisão dessas duas metodologias. Coward ${ }^{12}$, todavia, não encontrou um viés significativo entre elas.

O enriquecimento isotópico das amostras é analisado pela espectrometria de massa por determinação da razão isotópica (IRMS), método analítico que determina as massas com extrema rapidez, precisão, especificidade e sensibilidade ${ }^{1}$.

\section{Validade e precisão}

Em humanos, a precisão do método em calcular a produção de $\mathrm{CO}_{2}$ varia entre $93 \%$ a $97 \%$, dependendo das condições do experimento e do estado fisiológico dos sujeitos ${ }^{4}$. A acurácia do método é de $97 \%$ a $99 \%$ em relação à calorimetria indireta 5 . Em estudos com adultos em balanço energético, nos quais os resultados provenientes da água duplamente marcada foram comparados com outros obtidos por meio da calorimetria indireta, Coward et al..$^{13}$ obteveram erro de $1,9 \pm 2 \%$ na estimativa da produção do $\mathrm{CO}_{2}$, enquanto que Schoeller \& Webb' ${ }^{14}$ observaram erro de 1,5 $\pm 7,6 \%$. De acordo com
Roberts ${ }^{4}$, as mensurações são tão acuradas em adultos quanto em crianças, indicando que a perda de água devido à lipogênese de novo, que normalmente ocorre durante períodos de rápido crescimento, não constitui uma fonte de erro. A utilização da metodologia de dois pontos ${ }^{5}$ diminui a precisão da água duplamente marcada em $1 \%$ a $2 \%$.

\section{Aplicações}

Sendo a água duplamente marcada o método mais acurado de mensuração do gasto energético total em indivíduos fora de confinamento, suas aplicações são inúmeras. Pode-se, contudo, dividir a utilidade da técnica em quatro tópicos: no estudo da etiologia da obesidade; na determinação do gasto energético em condições especiais, como lactação, práticas esportivas e condições clínicas; na validação de instrumentos de avaliação da prática de atividades físicas e/ou do gasto energético; e na validação dos métodos de avaliação do consumo alimentar.

\section{Estudo da obesidade}

Antes da água duplamente marcada tornar-se uma técnica viável em estudos com humanos, acreditava-se que os indivíduos obesos possuíam gasto energético inferior aos eutróficos. Especulava-se que os obesos seriam mais "eficientes" em termos de metabolismo energético, e que essa seria a causa do ganho de peso. Em 1986, entretanto, Prentice et al. ${ }^{15}$ realizaram estudo pioneiro, demonstrando que as mulheres obesas tinham gasto energético maior que as eutróficas (gasto energético médio de $2445 \mathrm{kcal}$ e $1911 \mathrm{kcal}$, respectivamente, $p=0,001)$. Ao combinar essa técnica com a calorimetria indireta, foi descoberto que tal aumento era proveniente do incremento na taxa de metabolismo basal e na demanda energética durante a prática de exercícios. Ao controlar tais resultados pela massa magra e peso corporal, os autores constataram que o gasto energético das obesas era semelhante ao das 
eutróficas. Esses achados foram corroborados mais tarde pela meta-análise, feita por Prentice et al. ${ }^{16}$, de 319 mensurações do gasto energético pela água duplamente marcada. Buhl et al. ${ }^{17}$ avaliaram dez pacientes com histórico de distúrbios na regulação do peso corporal, com o intuito de identificar se eles apresentavam baixo gasto energético que justificasse tal distúrbio. Todos os pacientes relatavam baixo consumo energético. Eles foram comparados com obesos que não relatavam baixo consumo energético e distúrbios na regulação do peso. Os dez indivíduos apresentaram concentrações séricas de hormônios tireoidianos normais. Oito pacientes apresentavam taxa metabólica basal e gasto energético normais. Um apresentou baixo gasto energético e taxa de metabolismo basal normal, indicando estilo de vida sedentário. Apenas um paciente obteve o gasto energético e a taxa de metabolismo basal baixos, indicando de fato a presença de distúrbio.

Alguns estudos foram conduzidos para investigar o papel do gasto energético total na diferente prevalência de obesidade entre os grupos étnicos. Wong et al. ${ }^{18}$, ao estudar oitenta e uma adolescentes, encontraram que as afro-americanas despendiam menos energia do que as brancas, tanto no repouso quanto durante a prática de exercícios. Weinsier et al. ${ }^{19}$ conduziram estudo bastante interessante, no qual mulheres negras e brancas, com sobrepeso, foram submetidas a dietas restritivas, visando a perda de peso. Antes e depois da intervenção, foram realizadas mensurações do gasto energético total (por água duplamente marcada) e do gasto durante o sono, repouso e exercício (por calorimetria indireta). Após a perda de peso, houve redução no gasto energético durante o sono e o repouso. As negras apresentaram menor gasto energético (antes e depois da perda de peso) durante o sono, repouso e exercício, assim como o gasto total. Entretanto, a prática de atividade física foi similar entre as negras e brancas, o que, combinado com o menor gasto, pode predispor as negras ao reganho de peso.

Poucos estudos longitudinais a esse respeito foram desenvolvidos. Stunkard et al. ${ }^{20}$ mediram o gasto energético pela água duplamente marcada em quarenta crianças filhas de mães obesas e trinta e oito de mães eutróficas, aos três meses de idade. O gasto energético não foi capaz de prever o peso corporal ao completar um ano de idade. Goran et al. ${ }^{21}$ acompanharam, por quatro anos, setenta e cinco adolescentes brancos, realizando semestralmente administrações de água duplamente marcada. Eles não observaram influência do gasto energético na taxa de ganho de massa gorda/ano, sendo esta ajustada pela massa magra ou não. Schoeller et al. ${ }^{22}$ acompanharam por um ano adultas que tivessem emagrecido e atingido seu peso ideal nos últimos três meses. O gasto energético foi medido por meio da água duplamente marcada, e a taxa de metabolismo basal pela calorimetria indireta. A razão gasto energético total/taxa de metabolismo basal constitui indicador da prática de atividade física. Os autores constataram que quanto maior esse indicador, menor era o reganho de peso, e concluíram que um gasto mínimo de 47kilojoules/kg de peso corporal por dia poderia prevenir a recuperação do peso corporal.

Embora o pequeno número de estudos, especialmente os longitudinais, dificulte a formulação de hipóteses, pode-se supor que a contribuição do gasto energético para a etiologia da obesidade varie conforme a idade. A comparação entre os achados é problemática, posto que se deve ajustar o gasto energético pelo peso e composição corporais. Todavia, as evidências citadas anteriormente parecem sugerir que o alto consumo energético, e não o gasto, esteja causando desequilíbrio no balanço energético.

\section{Determinação do gasto energético em condições especiais}

A mensuração do gasto energético durante a prática de atividade física é importante para determinar as necessidades energéticas de seus praticantes, mas muitas vezes isso não é possível posto que a calorimetria indireta implica o confinamento dos sujeitos. Assim, a água duplamente 
marcada obteve êxito ao avaliar o gasto energético em modalidades nas quais é impossível o confinamento, como na prova de ciclismo Tour de France ${ }^{23}$, no nado sincronizado ${ }^{24}$, no iatismo ${ }^{25}$ e no alpinismo ${ }^{26}$. A água duplamente marcada também foi aplicada para mensuração do efeito do treinamento e do repouso forçado sobre o gasto de energia. Blanc et al. ${ }^{27}$ notaram redução de $20 \%$ do dispêndio energético durante o período de repouso absoluto. Eliakim et al. ${ }^{28}$ demonstraram que, ao cabo de cinco semanas de treinamento, adolescentes do sexo masculino apresentavam gasto $15 \%$ superior ao dos seus controles sedentários. Hayes et al. ${ }^{29}$ conduziram estudo muito interessante, no qual os sujeitos foram submetidos ao transplante periférico de células tronco do sangue, seguido de três meses de atividades físicas moderadas. O gasto energético foi medido pela água duplamente marcada nos momentos pré- e pós- transplante e após três meses de exercícios. O transplante não provocou mudanças no gasto energético, enquanto que a prática de atividade física o aumentou.

Borgonha et al. ${ }^{30}$ compararam o gasto energético de três grupos de indianos: a) sujeitos com índice de massa corporal (IMC) normal, que viviam em cidades; b) sujeitos com IMC normal, que viviam no meio rural e; c) sujeitos com IMC abaixo do normal, que viviam em cidades. O grupo b apresentou o maior gasto energético, indicando, provavelmente, a realização de intenso trabalho manual por parte deles. O grupo c obteve o menor gasto e nível de atividade física, o que pode consistir em uma estratégia de conservação da energia, para manutenção do peso corporal. Hoffman et al. ${ }^{31}$ utilizaram a água duplamente marcada para medir o gasto de energia de crianças moradoras de favelas na cidade de São Paulo. Foi testada a hipótese de as crianças desnutridas apresentarem menor gasto energético, o que, tardiamente, poderia ser fator de risco para obesidade. Não foram encontradas diferenças entre as crianças desnutridas e aquelas com peso normal, quando os resultados foram controlados quanto ao peso e composição corporais. $\mathrm{Na}$ Guatemala, Wren et al. ${ }^{32}$ também não observaram diferenças de gasto energético entre crianças com baixa estatura e crianças com estatura normal. Os autores afirmaram que havia grande variação entre o dispêndio energético de cada criança, que não podia ser explicada apenas pelo peso e composição corporais. Slinde et al. ${ }^{33}$ mensuraram o gasto energético de pacientes com doença pulmonar obstrutiva crônica, que apresentavam baixo peso e não estavam internados. O gasto energético variou de 110 a 200 kilojoules/kg de peso corporal. Devido a essa grande variação, os autores concluíram que não seria correto estimar o dispêndio energético desses pacientes a partir de equações.

Percebe-se, portanto, que o gasto energético pode variar imensamente de acordo com a idade, sexo, condições de vida, peso, composição corporal, prática de atividade física e estado fisiológico. É de suma importância que se conduzam estudos com água duplamente marcada em diversas populações, a fim de avaliar suas necessidades e determinar suas recomendações de consumo energético.

\section{Validação de métodos de avaliação da prática de atividade física e/ou do gasto energético}

A água duplamente marcada é uma técnica de alto custo e que requer equipamentos sofisticados e pessoal amplamente treinado. Assim, sua aplicação em grandes estudos, especialmente os epidemiológicos, torna-se, muitas vezes, inviável. No intuito de preencher essa lacuna, alguns estudos validaram métodos de avaliação da prática de atividade física e/ou do gasto energético, confrontando esses resultados com outros obtidos pela água duplamente marcada. Na grande maioria dos estudos, a mensuração do gasto energético por essa técnica foi combinada com a determinação da taxa de metabolismo basal pela calorimetria indireta. Subtraindo-se a taxa de metabolismo basal e o efeito térmico dos alimentos do gasto energético, tem-se o gasto energético durante a prática de 
atividades físicas, que é a variável comparada entre a água duplamente marcada e as outras técnicas.

Livingstone et al. ${ }^{34}$ compararam o gasto energético obtido pela água duplamente marcada com o obtido pelo monitoramento da freqüência cardíaca em crianças e adolescentes. Os autores encontraram, em média, diferença percentual de $-1,5 \pm 13,4$ para crianças de sete anos; $-9,2 \pm 4,5$ para crianças de nove anos; $-1,5 \pm 7,1$ para crianças com doze anos e $3,5 \pm 6,6$ para aquelas com quinze anos. Foi concluído que o monitoramento da freqüência cardíaca fornecia estimativa próxima do gasto energético, e uma medida objetiva da prática de atividade física. Lovelady et al. ${ }^{35}$ realizaram a validação do monitoramento da freqüência cardíaca com nutrizes. Embora a diferença percentual entre os dois métodos tenha sido 5,8\%, em média, a diferença individual variou de $-27,1 \%$ a $17,6 \%$. Dessa forma, embora a monitoração da freqüência possa fornecer estimativas médias razoáveis, ela é insatisfatória em nível individual. Johnson et al. ${ }^{36}$ compararam o gasto durante a prática de atividades físicas obtido pela água duplamente marcada com outro, obtido por um acelerômetro, que media o deslocamento do centro de gravidade em três eixos. A concordância entre os dois foi fraca e a movimentação registrada pelo acelerômetro praticamente não explicou a variação obtida no gasto energético despendido durante atividades físicas. Ekelund et al. ${ }^{37}$ encontraram $r=0,67(p<0,001)$ entre a contagem de movimentos por um acelerômetro de eixo vertical e o gasto energético total pela água duplamente marcada. Os autores também desenvolveram uma equação para calcular o gasto energético a partir da contagem de movimentos. A diferença entre o gasto mensurado pela água duplamente marcada e o predito por essa equação não foi significativa, variando entre -23 a $-69 \mathrm{kcal} / \mathrm{dia}$, dependendo das variáveis inclusas na equação. Entretanto, os limites do intervalo de $95 \%$ de concordância foram muito díspares e amplos, mostrando que a comparação individual entre os métodos não foi adequada. Assim, a equação desenvolvida é válida apenas quando aplicada para estimar o gasto energético médio de grupos. Lof et al. ${ }^{38}$ compararam o gasto energético obtido pela água duplamente marcada com outros obtidos pelo monitoramento da freqüência cardíaca, acelerômetro e questionário de atividade física, em mulheres em idade reprodutiva. Todas as técnicas superestimaram o gasto, sendo que a diferença entre o gasto medido pela água marcada e pelas outras técnicas foi de $58 \pm 338 \mathrm{kcal} / \mathrm{dia}$ (para o monitoramento da freqüência), $6 \pm 325 \mathrm{kcal} / \mathrm{dia}$ (para o acelerômetro) e $204 \pm 508 \mathrm{kcal} / \mathrm{dia}$ (para o questionário).

Conway et al. ${ }^{39}$ compararam o gasto energético determinado pela água duplamente marcada com as estimativas obtidas por um registro da prática de atividade física, preenchido durante sete dias, e por recordatório da prática de exercícios nos sete dias anteriores. Os autores concluíram que o registro é um método válido para estimar a prática de atividade física e, subseqüentemente, o gasto energético, ao contrário do recordatório, que superestimou ambas as variáveis. Philippaerts et al. ${ }^{40}$ compararam os resultados de três questionários de prática de atividade física com mensurações de água duplamente marcada e concluíram que ambos eram válidos e úteis em estudos epidemiológicos, especialmente o questionário de Baecke. Bonnefoy et al. ${ }^{41}$ validaram simultaneamente dez questionários de prática de atividade física em idosos. O Seven Day Recall e o Stanford Usual Activity Questionnaire foram os únicos que forneceram médias acuradas. Esse último também teve boa correlação com o consumo máximo de oxigênio.

Dessa forma, percebe-se que há enorme necessidade de desenvolver métodos válidos, porém mais simples e acessíveis, para cálculo do gasto energético e avaliação da prática de atividade física. Embora algumas técnicas, em especial os questionários e os acelerômetros, sejam capazes de fornecer estimativas a respeito da prática de atividade física, elas não são suficientemente válidas para estimar o gasto energético. Todos os métodos apresentados 
podem obter estimativas médias razoáveis, mas, quando aplicados a indivíduos, seu desempenho é insatisfatório.

\section{Validação dos métodos de avaliação do consumo alimentar}

Embora, há muito tempo, já se soubesse que cada método de avaliação do consumo alimentar apresenta suas limitações, a quantificação dos erros conseqüentes de tais limitações e a posterior validação dos métodos não eram factíveis até recentemente ${ }^{42}$. Muitos estudos realizaram a validação relativa (ou calibração, como preferem alguns autores) dos métodos de inquérito alimentar, o que consistia na comparação das estimativas obtidas por cada método. Contudo, foi descoberto que os métodos utilizados como referência nos estudos de validação relativa também estavam sujeitos aos mesmos erros sistemáticos do instrumento que estava sendo testado ${ }^{43}$. Aplicando-se a água duplamente marcada, constatou-se que a ingestão energética relatada nas avaliações de consumo alimentar era, freqüentemente, bem menor do que a real. Tal fenômeno foi denominado underreporting of energy intake, ou, em português, sub-relato ou subnotificação da ingestão energética. Em indivíduos em balanço energético, a ingestão de energia deve ser igual ao seu gasto. Assim, a água duplamente marcada pode ser utilizada para validar o consumo de energia obtido por meio dos inquéritos alimentares ${ }^{44}$.

Utilizando a água duplamente marcada, Goris et al. ${ }^{45}$ encontraram sub-relato de $37 \pm 16 \%$ da ingestão energética em homens obesos, sendo que a maior parte desse correspondia ao subconsumo, isto é, à diminuição do consumo alimentar causada pelo próprio instrumento de avaliação. Sawaya et al. ${ }^{46}$ compararam os resultados obtidos pela água duplamente marcada com as estimativas de ingestão energética fornecidas por diários alimentares de sete dias (com pesagem das porções), recordatórios de vinte e quatro horas e questionários de freqüência alimentar. Foi concluído que nenhum método fornecia estimativas individuais válidas, sendo que o diário alimentar teve o pior resultado.

Perks et al. ${ }^{47}$ observaram, em pré-adolescentes, que os limites de concordância \pm 2 desvios-padrão entre o gasto energético (pela água marcada) e a ingestão de energia, estimada por um questionário de freqüência alimentar, eram muito amplos, embora o consumo de energia tenha sido semelhante ao gasto. Quando se analisou a acurácia entre os indivíduos, por meio dos coeficientes de variação intra-individual, encontrou-se que $50 \%$ da amostra havia sub-relatado a ingestão energética. Herbert et al. ${ }^{48}$ avaliaram o efeito da aprovação social e desejo de aceitação social na distorção das estimativas de ingestão energética obtidas por sete recordatórios de 24 horas, um diário alimentar de sete dias e um questionário de freqüência alimentar, de acordo com a água duplamente marcada. Entre as mulheres com curso superior, o desejo de aceitação social teve correlação negativa com a ingestão energética estimada pelo questionário de freqüência. Entre as mulheres com menor escolaridade, a aprovação social causou superestimação da ingestão. Naquelas com curso superior, o efeito foi inverso.

A água duplamente marcada também tem sido aplicada para validar as novas tecnologias aplicadas aos métodos de avaliação do consumo. Tran et al. ${ }^{49}$ compararam recordatórios alimentares de vinte e quatro horas aplicados pessoalmente e pelo telefone. Os dois foram igualmente efetivos, porém essa efetividade foi baixa, posto que o sub-relato estava altamente presente em ambas as formas de aplicação. Lindquist et al..$^{50}$ analisaram, em crianças, a validade de três recordatórios de vinte e quatro horas e de três dias de diário alimentar, gravados pelos sujeitos em fita cassete, em vez de escritos manualmente. O sub-relato esteve presente em $43 \%$ dos recordatórios e $61 \%$ dos diários. A superestimativa, por sua vez, foi encontrada em $40 \%$ dos recordatórios e $26 \%$ dos diários. O novo método se mostrou válido apenas em crianças mais novas e de menor peso. 
Kaczkowski et al. ${ }^{51}$ combinaram o uso de gravador de áudio com câmera fotográfica. A acurácia do relato foi de $76 \pm 22,9 \%$, indicando intenso sub-relato. Vários aspectos do sub-relato permanecem largamente inexplorados, comprometendo de forma significativa as inferências feitas a partir de estudos de avaliação do consumo alimentar. A interpretação desses, portanto, deve ser feita com cautela. A utilização da água duplamente marcada como instrumento de validação dos métodos de avaliação do consumo alimentar é altamente recomendável. Tal procedimento deve ser implementado em estudos de países em desenvolvimento, nos quais, embora nenhum estudo de validação com água duplamente marcada tenha sido desenvolvido, diversos levantamentos populacionais do consumo alimentar são conduzidos. Usualmente, é assumido que os métodos de avaliação do consumo que foram validados em outros países também sejam válidos no Brasil, o que pode não ser verdadeiro. Além disso, não se conhece o comportamento do sub-relato no Brasil. Sendo esse fenômeno altamente influenciado por fatores sociais e culturais, é importante estudá-lo a priori, em qualquer população do qual se pretende mensurar consumo.

\section{O N C L U S Ã O}

A mensuração acurada do gasto energético é de suma importância em estudos de nutrição, o que deve fazer da água duplamente marcada uma técnica amplamente empregada. Constata-se grande variação no gasto energético, ocasionada pela idade, sexo, grupo étnico, peso, composição corporal, prática de atividades físicas, condições de saúde e estado fisiológico. Assim, a estimativa do gasto energético por equações pode não ser suficiente nos casos em que tais fatores possam causar grande variação no gasto energético. Em tais casos, a aplicação da água duplamente marcada permite melhor avaliação do gasto energético e seus desdobramentos, como as necessidades energéticas. Por fim, a água duplamente marcada deve ser utilizada como marcador em estudos de avaliação do consumo alimentar, posto que, para cada população estudada, é necessário determinar a validade do instrumento de avaliação.

\section{A GRADECIMENTOS}

Os autores agradecem à colega Viviane Ozores Polacow, pela cuidadosa revisão, e ao CNPq e à FAPESP (processo 03/12337-8), pelo apoio financeiro.

\section{REFERÊ NCIAS}

1. Speakman JR. The history and theory of the doubly labeled water technique. Am J Clin Nutr. 1998; 68(4):5932-8

2. Lifson N, Gordon GB, Visscher MB, Nier AO. The fate of utilized molecular oxygen and the source of the oxygen of respiratory carbon dioxide, studied with the aid of heavy oxygen. J Biol Chem. 1949; 180:803-11.

3. Lifson N, Gordon GB, Mcclintock R. Measurement of total carbon dioxide production by means of D2180. J Appl Physiol. 1955; 7(6):704-10.

4. Roberts S. Use of the doubly labeled water method for measurement of energy expenditure, total body water, water intake, and metabolizable energy intake in humans and small animals. Can J Physiol Pharmacol. 1989; 67(10):1190-8.

5. Nagy KA. Introduction. In: Prentice AM, editor. The doubly labelled water method for measuring energy expenditure. Technical recomendations for use in humans. Vienna: International Dietary Energy Consultancy Group; 1990. p.1-16.

6. Schoeller DA, Leitch CA, Brown C. Doubly labeled water method: in vivo oxygen and hydrogen isotope fractionation. Am J Physiol. 1986; 20(6 Pt 2):1137-43.

7. Weir JB. New methods for calculating metabolic rate with special reference to protein metabolism. J Psysiol. 1949; 109 (1-2):1-9.

8. Elia M. Converting carbon dioxide production to energy expenditure. In: Prentice AM, editor. The doubly labelled water method for measuring energy expenditure. Technical recomendations for use in humans. Vienna: International Dietary Energy Consultancy Group; 1990. p.193-211.

9. Black $A E$, Prentice AM, Coward WA. Use of food quotients to predict respiratory quotients for the doubly-labelled water method of measuring 
energy expenditure. Hum Nutr Clin Nutr. 1986; 40(5):381-91.

10. Southgate DAT, Durnin JVGA. Calorie conversion factors. An experimental reassessment of the factors used in the calculation of the energy value of human diets. Br J Nutr. 1970; 24(2):517-35.

11. Coward WA. Calculation of pool sizes and flux rates. In: Prentice AM, editor. The doubly-labelled-water method for measuring energy expenditure. Technical recomendations for use in humans. Vienna: International Dietary Energy Consultancy Group; 1990. p. 48-68.

12. Coward WA. The doubly-labelled-water (2H2180) method: principles and practice. Proc Nutr Soc. 1988; 47(3):209-18.

13. Coward WA, Prentice AM, Murgatroyd PR, Davies $\mathrm{HL}$, Cole TJ, Sawyer M, et al. Measurements of $\mathrm{CO}_{2}$ and water production rates in man using ${ }^{2} \mathrm{H}^{18} \mathrm{O}$; comparisons between calorimeter and isotopes values. In: Van Es AJH. A concerned action project on nutrition in the European Community, Wageningen: Agricultural University; 1984.

14. Schoeller DA, Webb P. Five-day comparison of the doubly labeled water method with respiratory gas exchange. Am J Clin Nutr. 1984; 40(1):153-8.

15. Prentice AM, Black AE, Coward WA, Davies $H L$, Goldberg GR, Murgatroyd PR, et al. High levels of energy exependiture in obese women. BMJ. 1986; 292(6526):983-7.

16. Prentice AM, Black AE, Coward WA, Cole TJ. Energy expenditure in overweight and obese adults in affluent societs: an analysis of 319 doubly labelled water measurements. Eur J Clin Nutr. 1996; 50(2):93-7.

17. Buhl KM, Gallagher D, Hoy K, Matthews DE, Heymsfield SB. Unexplained disturbance in body weight regulation: Diagnostic outcome assessed by doubly labeled water and body composition analyses in obese patients reporting low energy intakes. J Am Diet Assoc. 1995; 95(12):1393-400.

18. Wong WW, Butte NF, Ellis KJ, Herenroader AC, Hill RB, Stuff JE, et al. Pubertal African-Americans girls expend less energy at rest and during physical activity than Caucasian girls. J Clin Endocrinol Metab. 1999; 84(3):906-11.

19. Weinsier RL, Hunter GR, Zuckerman PA, Redden DT, Darnell BE, Larsson DE, et al. Energy expenditure and free-living physical activity in black and white women: comparison before and after weight loss. Am J Clin Nutr. 2000; 71(5):1138-46.

20. Stunkard AJ, Berkowitz RI, Stallings VA, Schoeller DA. Energy intake, not energy output, is a determinant of body size in infants. Am J Clin Nutr. 1999; 69(3):524-30.
21. Goran MI, Shewchuk R, Gower BA, Nagy TR, Carpenter WH, Johnson RK. Longitudinal changes in fatness in white children: no effect of childhood energy expenditure. Am J Clin Nutr. 1998; 67(2):309-16.

22. Schoeller DA, Shay K, Kushner RF. How much physical activity is needed to minimize weight gain in previously obese women? Am J Clin Nutr. 1997; 66(3):551-6.

23. Westerterp KR, Saris WHM, Van Es M, Ten Hoor F. Use of the doubly labeled water technique in humans during heavy sustained exercise. J Appl Physiol. 1986; 61(6):2161-7.

24. Ebine N, Feng JY, Homma M, Saitoh S, Jones PJ. Total energy expenditure of elite synchronized swimmers measured by the doubly labeled water method. Eur J Appl Physiol. 2000; 83(1):1-6.

25. Branth S, Hambreus L, Westerterp K, Anderssen A, Edsgren $\mathrm{R}$, Mustelin $\mathrm{M}$, et al. Energy turnover in a sailing crew during off-shore racing around the world. Med Sci Sports Exerc. 1996; 28(10): 1272-6.

26. Pulfrey SM, Jones PJ. Energy expenditure and requirement while climbing above 6,000m. J Appl Physiol. 1996; 81(3):1306-11.

27. Blanc S, Normand S, Ritz P, Pachiaudi C, Vico L, Gharib C, et al. Energy and water metabolism, body composition, and hormonal changes induced by 42 days of enforced inactivity and simulated microgravity. J Clin Endocrinol Metab. 1998; 83(12):4289-97.

28. Eliakim A, Brasel JA, Mohan S, Wong WLT, Cooper DM. Increased physical activity and the growth hormone IGF-I-axis in adolescents. Am J Physiol. 1998; 275(1 Pt 2):R308-14.

29. Hayes S, Davies PS, Parker T, Bashford J. Total energy expenditure and body composition changes following peripheral blood stem cell transplantation and participation in an exercise program. Bone Marrow Transplant. 2003; 31(5):331-8.

30. Borgonha S, Shetty PS, Kurpad AV. Total energy expenditure \& physical activity level in chronically energy deficient Indian males measured by the doubly labelled water technique. Ind J Med Res. 2000; 111:138-46.

31. Hoffman DJ, Sawaya AL, Coward WA, Wright A, Tucker K, Roberts SB. Energy expenditure of stunded and nonstunted boys and girls living in the shantytowns of São Paulo. Am J Clin Nutr. 2000; 72(4):1025-31.

32. Wren RE, Blume $H$, Mazariegos $M$, Solomons $N$, Alvarez JO, Goran MI. Body composition, resting metabolic rate, and energy requirements of 
short- and normal-stature, low-income Guatemalan children. Am J Clin Nutr. 1997; 66(2):406-12.

33. Slinde F, Ellegard L, Gronberg AM, Larsson S, Rossander-Hulthen L. Total energy expenditure in underweight patients with severe chronic obstructive pulmonary disease living at home. Clin Nutr. 2003; 22(2):159-65.

34. Livingstone MB, Coward WA, Prentice AM, Davies PS, Strain JJ, Mckenna PG, et al. Daily energy expenditure in free-living children: comparison of heart-rate monitoring with the doubly labeled water method. Am J Clin Nutr. 1992; 56(2): 343-52.

35. Lovelady CA, Meredith CN, McCrory MA, Nommsen LA, Joseph I, Dewey KG. Energy expenditure in lactating women: a comparison of doubly labeled water and heart rate monitoring methods. Am J Clin Nutr. 1993; 57(4):512-8.

36. Johnson RK, Russ J, Goran MI. Physical activity related energy expenditure in children by doubly labeled water as compared with the Caltrac accelerometer. Int J Obes Relat Metab Disord. 1998; 22(11):1046-52.

37. Ekelund U, Sjoström M, Yngve A, Poortvliet E, Nilsson A, Froberg K, et al. Physical activity assessed by a activity monitor and doubly labeled water in children. Med Sci Sports Exerc. 2001; 33(2): 275-81.

38. Lof M, Hannestad U, Forsum E. Assessing physical activity of women of childbearing age. Ongoing work to develop and evaluate simple methods. Food Nutr Bull. 2002; 23(3 Suppl):30-3.

39. Conway JM, Seale JL, Jacobs Jr DR, Irwin ML, Ainsworth BE. Comparison of energy expenditure estimates from doubly labeled water, a physical activity questionnaire, and physical activity records. Am J Clin Nutr. 2002; 75(3):519-25.

40. Philippaerts RM, Westerterp KR, Lefevre J. Doubly labelled water validation of three physical activity questionnaires. Int J Sports Med. 1999; 20(5): 284-9.

41. Bonnefoy M, Normand S, Pachiaudi C, Lacour JR, Laville M, Kostka T. Simultaneous validation of ten physical activity questionnaires in older man: a doubly labeled water study. JAGS. 2001; 49(1): 28-35.
42. Livingstone MBE, Black AE. Markers of the validity of reported energy intake. J Nutr. 2003; 133 Suppl 3:S895-920.

43. Scagliusi FB, Lancha Junior AH. Subnotificação da ingestão energética na avaliação do consumo alimentar. Rev Nutr. 2003; 16(4):471-81.

44. Bellisle F. The doubly-labeled-water method and food intake surveys: a confrontation. Rev Nutr. 2001; 14(2):125-33.

45. Goris AH, Westerterp-Plantenga MS, Westerterp $\mathrm{KR}$. Undereating and underrecording of habitual food intake in obese men: selective underreporting of fat intake. Am J Clin Nutr. 2000; 71(1):130-4.

46. Sawaya AL, Tucker K, Tsay R, Willett W, Saltzman E, Dallal GE, et al. Evaluation of four methods for determining energy intake in young and older women: comparison with doubly labeled water measurements of total energy expenditure. Am J Clin Nutr. 1996; 63(4):491-9.

47. Perks SM, Roemmich JN, Sandow-Pajewski M, Clark PA, Thomas E, Weltman A, et al. Alterations in growth and body composition during puberty. IV. Energy intake estimated by the Youth-Adolescent Food-Frequency Questionnaire: validation by the doubly labeled water method. Am J Clin Nutr. 2000; 72(6):1455-60.

48. Herbert JR, Ebbeling CB, Matthews CE, Hurley TG, Yunsheng MA, Druker $S$, et al. Systematic errors in middle-aged women's estimates of energy intake: comparing three self-reports measures to total energy expenditure from doubly labeled water. Ann Epidemiol. 2002; 12(8):577-86.

49. Tran KM, Johnson RK, Soultanakis RP, Matthews DE. In-person vs. telephone-administered multiple-pass 24-hour recall in women: validation with doubly labeled water. J Am Diet Assoc. 2000; 100(7):777-83.

50. Lindquist $\mathrm{CH}$, Cummings T, Goran MI. Use of taperecorded food records in assessing children's dietary intake. Obes Res. 2000; 8(1):2-11.

51. Kaczkowski CH, Jones PJ, Feng JY, Bayley HS. Four-day multimedia diet records underestimate energy needs in middle-aged and eldery women as determined by doubly labeled water. J Nutr. 2000b; 130(4):802-5.

Recebido para publicação em 27 de novembro de 2003 e aceito em 28 de outubro de 2004. 